\begin{tabular}{|c|l|}
\hline Title & Some special bounded homomorphisms of a uniform algebra \\
\hline Author(s) & Nakazi, T. \\
\hline Citation & Hokkaido University Preprint Series in Mathematics, 443, 1-10 \\
\hline Issue Date & 1999-2-1 \\
\hline DOI & 10.14943/83589 \\
\hline Doc URL & http://hdl.handle.net/2115/69193 \\
\hline Type & bulletin (article) \\
\hline File Information & pre443.pdf \\
\hline
\end{tabular}

Instructions for use 
Some Special Bounded Homomorphisms Of A Uniform

Takahiko Nakazi

Series \#443. February 1999 


\section{HOKKAIDO UNIVERSITY PREPRINT SERIES IN MATHEMATICS}

\#418 M. Ishikawa and S. Matsui, Existence of a forward self-similar stagnation flow of the Navier-Stokes equations, 8 pages. 1998.

\#419 S. Izumiya, H. Katsumi and T. Yamasaki, The rectifying developable and the spherical Darboux image of a space curve, 16 pages. 1998.

\#420 R. Kobayashi and Y. Giga, Equations with singular diffusivity, 45 pages. 1998.

\#421 D. Pei and T. Sano, The focal developable and the binormal indicatrix of a nonlightlike curve in Minkowski 3-space, 14 pages. 1998.

\#422 R. Kobayashi, J. A. Warren and W. C. Carter, Modeling grain boundaries using a phase field technique, 12 pages. 1998.

\#423 T. Tsukada, Reticular Legendrian Singularities, 28 pages. 1998.

\#424 A. N. Kirillov and M. Shimozono, A generalization of the Kostka-Foulkes polynomials, 37 pages. 1998.

\#425 M. Nakamura, Strichartz estimates for wave equations in the homogeneous Besov space, 17 pages. 1998.

\#426 A. Arai, On the essential spectra of quantum field Hamiltonians, 18 pages. 1998.

\#427 T. Sano, Bifurcations of affine invariants for one parameter family of generic convex plane curves, 11 pages. 1998.

\#428 F. Hiroshima, Ground states of a model in quantum electrodynamics, 48 pages. 1998.

\#429 F. Hiroshima, Uniqueness of the ground state of a model in quantum electrodynamics: A functional integral approach, 32 pages. 1998.

\#430 J. F. Van Diejen and A. N. Kirillov, Formulas for $q$-spherical functions using inverse scattering theory of reflectionless Jacobi operators, 33 pages. 1998.

\#431 G. Ishikawa, Determinacy, transversality and Lagrange stability, 13 pages. 1998.

\#432 T. Yoshida, Categorical aspects of generating functions(II): Operations on categories and functors, 65 pages. 1998.

\#433 K. Ito, Loss of convexity of compact hypersurfaces moved by surface diffusion, 20 pages. 1998.

\#434 Y. Shimizu, $L^{\infty}$-estimate of first-order space derivatives of Stokes flow in a half space, 22 pages. 1998.

\#435 T. Uemura, Morita-Mumford classes on finite cyclic subgroups of the mapping class group of closed surfaces, 14 pages. 1998.

\#436 A. Inoue and H. Kikuchi, Abel-Tauber theorems for Hankel and Fourier transforms and a problem of Boas, 20 pages. 1998.

\#437 T. Nakazi and T. Osawa, Finite rank intermediate Hankel operators on the Bergman space, 16 pages. 1998.

\#438 R. Yoneda, Compact Toeplitz operators on Bergman spaces, 14 pages. 1998.

\#439 A. Inoue and Y. Kasahara, On the asymptotic behavior of the prediction error of a stationary process, 12 pages. 1998.

\#440 A. Arai, M. Hirokawa and F. Hiroshima, On the absence of eigenvectors of Hamiltonians in a class of massless quantum field models without infrared cutoff, 26 pages. 1998.

\#441 A. N. Kirillov, A. Schilling and M. Shimozono, A bijection between Littlewood-Richardson tableaux and rigged configurations, 66 pages. 1999.

\#442 M. Tsujii, Absolutely continuous invariant measures for expanding piecewise linear maps, 28 pages. 1999. 


\title{
Some Special Bounded Homomorphisms Of A Uniform Algebra
}

\author{
Takahiko Nakazi
}

\begin{abstract}
Let $L(H)$ be the algebra of all bounded linear operators on a Hilbert space $H$ and let $A$ be a uniform algebra. In this paper we study the following questions. When is a unital bounded homomorphism $\Phi$ of $A$ in $L(H)$ completely bounded? When is the norm $\|\Phi\|$ of $\Phi$ equal to the completely bounded norm $\|\Phi\|_{c b}$ ? In some special cases we answer this question. Suppose $\Phi$ is $\rho$-contractive $(0<\rho<\infty)$ where $\Phi$ is contractive if $\rho=1$. We show that if $A$ is a Dirichlet algebra or $\operatorname{dim} A / \operatorname{ker} \Phi=2$ then $\Phi$ has a $\rho$-dilation. If $\Phi$ is a $\rho$-contractive homomorphism then $\|\Phi\|=\max (1, \rho)$ and if it has a $\rho$-dilation then $\|\Phi\|_{c b}=\max (1, \rho)$. Moreover we give a new example of a hypo-Dirichlet algebra in which a unital contractive homomorphism has a contractive dilation.
\end{abstract}

\section{Introduction}

Let $X$ be a compact Hausdorff space, let $C(X)$ be the algebra of complexvalued continuous functions on $X$, and let $A$ be a uniform algebra on $X$. Let $H$ be a complex Hilbert space and $L(H)$ the algebra of all bounded linear operators on $H . I=I_{H}$ is the identity operator in $H$. An algebra homomorphism $f \rightarrow \Phi(f)$ of $A$ in $L(H)$, which satisfies

$$
\Phi(1)=I \text { and }\|\Phi(f)\| \leq \gamma\|f\|_{\infty}
$$

for some positive constant $\gamma \geq 1$, is called a unital bounded homomorphism of $A$. If $\gamma=1$, it is called a unital contractive homomorphism.

For a subspace $B$ of $A$, let $M_{n}(B)$ denote the set of $n \times n$ matrices with entries from $B$. For a map $\phi: B \rightarrow L(H)$, we obtain maps $\phi_{n}: M_{n}(B) \rightarrow M_{n}(L(B))$ via the formula

$$
\phi_{n}\left(\left(a_{i j}\right)\right)=\left(\phi\left(a_{i j}\right)\right) .
$$

If $\phi$ is a bounded map, then each $\phi_{n}$ will be bounded, and when $\sup _{n}\left\|\phi_{n}\right\|$ is finite, we call $\phi$ a completely bounded map of $B$ in $L(H)$. We write

$$
\|\phi\|_{c b}=\sup _{n}\left\|\phi_{n}\right\| \text {. }
$$

The following problem is natural and important.

C0000 (copyright holder)

1991 Mathematics Subject Classification. Primary 47A20, 46J25.

The author was supported in part by Grant-in-Aid for Scientific Research, Ministry of Education. 
Problem . Suppose $\Phi$ is a unital bounded homomorphism of $A$.

I. When is $\Phi$ completely bounded?

II. When is $\Phi$ completely bounded and $\|\Phi\|=\|\Phi\|_{c b}$ ?

A unital contractive homomorphism $v \rightarrow \tilde{\Phi}(v)$ of $C(X)$ on a Hilbert space $K$ is called a contractive dilation of the unital contractive homomorphism $f \rightarrow \Phi(f)$ of $A$ on $H$ if $H$ is a Hilbert subspace of $K$ and

$$
\Phi(f)=P \tilde{\Phi}(f) \mid H \quad(f \in A)
$$

where $P$ is the orthogonal projection of $K$ onto $H$. If $\Phi$ has a contractive dilation then $\Phi$ is completely contractive and hence $\|\Phi\|=\|\Phi\|_{c b}$. If $\Phi$ is completely contractive then $\Phi$ has a contractive dilation. This is well known (see [18, Corollary 6.7]).

If $A$ is a uniform algebra and the uniform closure of $A+\bar{A}$, that is, $[A+\bar{A}]$ has finite codimension $n$ in $C(X)$ then $A$ is called a $n$-hypo-Dirichlet algebra and it is called a Dirichlet algebra when $[A+\bar{A}]=C(X)$, that is, $n=0$.

If $\operatorname{dim} H<\infty, \Phi$ is completely bounded for arbitrary uniform algebra $A$ (see [18, Exercises 3.11]). If $\operatorname{dim} H=\infty$ and $A$ is the disc algebra, then there exists a unital bounded homomorphism $\Phi$ which is not completely bounded. This was recently shown by G.Pisier [19]. If $A$ is a $n$-hypo-Dirichlet algebra and $\Phi$ is a unital contractive homomorphism then $\Phi$ is completely bounded. This was shown by R.G.Douglas and V.I.Paulsen [6]. However we don't know whether $\Phi$ is completely contractive or not. They are known solutions for Problem I.

Now we will give known solutions for Problem II when $\Phi$ is contractive. If $A$ is the disc algebra then there exists a contractive dilation. This is a famous theorem of B.Sz.-Nagy [10]. T.Ando [2] generalized this to the bidisc algebra. However S.K.Parrot [17] gave an example of $\Phi$ which does not have a contractive dilation in the polydisc algebra for $n \geq 3$. If $A$ is a Dirichlet algebra then there exists a contractive dilation (cf. [7]). For a $n$-hypo-Dirichlet algebra with $n \neq 0$, we don't know whether there exists a contractive dilation or does not. The polydisc algebra for $n \geq 2$ is not a $n$-hypo-Dirichlet algebra. If $A$ is an annulus algebra, that is, a rational function algebra on an annulus, then there exists a contractive dilation. This was shown by J.Agler [1]. An annulus algebra is a 1-hypo-Dirichlet algebra. If $\mathcal{A}$ is the disc algebra and $A=\{f \in \mathcal{A} ; f(0)=f(1)\}$, then $A$ is also a 1-hypoDirichlet algebra. The author [12] proved that $\Phi$ has a contractive dilation for this example. Even if $\operatorname{dim} H<\infty$, by an example of S.K.Parrot [17] $\Phi$ may not have a contractive dilation for some uniform algebra. The author and the late K.Takahashi [14], and Che-Chen Chu [5] showed that if $\operatorname{dim} H \leq 2, \Phi$ has a contractive dilation for an arbitrary uniform algebra.

Now we will give more concrete problems than Problem II.

PROBLEM . Suppose $\Phi$ is a unital bounded homomorphism of $A$.

(II-a) Suppose $\|\Phi\| \leq 1$. When $A$ is a $n$-hypo-Dirichlet algebra and $n \geq 1$, does $\Phi$ have a contractive dilation?

(II- $b$ ) Under what conditions on $\Phi$ which is $\|\Phi\|>1$, is $\Phi$ completely bounded with $\|\Phi\|=\|\Phi\|_{c b}$ when $A$ is a $n$-hypo-Dirichlet algebra and $n \geq 0$, or $\operatorname{dim} H \leq 2$ ?

In this paper, we study Problem (II- $a$ ) and (II- $b$ ). In $\S 2$, we give a new example of a 1-hypo-Dirichlet algebra in which a unital contractive homomorphism has a contractive dilation. In $\S 3$, we define a $\rho$-contractive homomorphism $\Phi$ and a $\rho$ dilation of $\Phi$ for $0<\rho<\infty$. If $\Phi$ is a $\rho$-contractive homomorphism then $\|\Phi\| \leq$ 
$\max (1, \rho)$ and if it has a $\rho$-dilation then $\|\Phi\|_{c b} \leq \max (1, \rho)$. In $\S 4$, we introduce a $\delta$-homomorphism of $A$ for $-\infty<\delta<1$. This homomorphism is bounded. In fact, we show more, that is, ' $\delta$-homomorphism' is equivalent to ' $\rho=1 /(1-\delta)$ contractive homomorphism'. In $\S 5$, we show that a $\rho$-contractive homomorphism has a $\rho$-dilation when $A$ is a Dirichlet algebra. In $\S 6$, we consider Problem II under conditions on $\Phi$, that is, $\operatorname{dim} A / \operatorname{ker} \Phi=2$ or a hypothesis on $\operatorname{ker} \Phi$.

\section{Third example of a hypo-Dirichlet algebra for Problem II-a}

For a $n$-hypo-Dirichlet algebra with $n \neq 0$, we know only two examples ([1], [11]) in which a unital contractive homomorphism has a contractive dilation, that is, Problem (II- $a$ ). They are 1-hypo-Dirichlet algebras. In this section, we give a new example which is also a 1-hypo-Dirichlet algebra. In the proof of Theorem 2.1, a theorem of T.Ando [2] is used essentially. Unfortunately we could not generalize Theorem 2.1 to $A=\left\{f \in \mathcal{A} ; f^{\prime}(0)=f^{\prime \prime}(0)=\cdots=f^{(n)}(0)=0\right\}$.

THEOREM 2.1. Let $\mathcal{A}$ be the disc algebra and $A=\left\{f \in \mathcal{A} ; f^{\prime}(0)=0\right\}$. If $\Phi$ is a unital contractive homomorphism of $A$ then $\Phi$ has a contractive dilation or equivalently $\Phi$ is a completely contractive.

Proof. Since $A=\mathbf{C}+z^{2} \mathcal{A}, A_{0}=\{f \in A ; f(0)=0\}=z^{2} \mathcal{A}$. $A_{0}$ has two generators, that is, $A_{0}$ is generated by $z^{2}$ and $z^{3}$ beause $2 \ell \pm 1$ can be written as the form $2 n+3 m$. Let $\Phi\left(z^{2}\right)=S$ and $\Phi\left(z^{3}\right)=T$ then $S T=T S,\|S\| \leq 1$ and $\|T\| \leq 1$. By a well known theorem of T.Ando [2], there exist two commuting operators $U$ and $V$ on a Hilbert space $K$ with $H \subset K$ such that

$$
S^{n} T^{m}=P U^{n} V^{m} \mid H
$$

for all nonnegative integers $n$ and $m$ where $P$ is an orthogonal projection from $K$ to $H$. Any polynomial $f$ in $A_{0}$ is written as the following:

$$
f=a_{10} z^{2}+a_{01} z^{3}+\sum_{j, \ell \geq 1} a_{j \ell} z^{2 j} z^{3 \ell}
$$

and so

$$
\begin{aligned}
\Phi(f) & =a_{10} S+a_{01} T+\sum_{j, \ell \geq 1} a_{j \ell} S^{j} T^{\ell} \\
& =P\left(a_{10} U+a_{01} V+\sum_{j, \ell \geq 1} a_{j \ell} U^{j} V^{\ell}\right) \mid H
\end{aligned}
$$

By a theorem of C.R.Putnam and B.Fuglede [20, Corollary 1.19], $U^{*} V=V U^{*}$. Hence if we set $\tilde{\Phi}\left(\left(z^{2}\right)^{j}\left(z^{3}\right)^{\ell}\right)=U^{j} V^{\ell}$ for any integers $j$ and $\ell$ then $\tilde{\Phi}$ is a unital contractive homomorphism of $C(X)$ in $L(K)$ and $\Phi=P \tilde{\Phi} \mid H$ on $A_{0}$. Thus $\tilde{\Phi}$ is a contractive dilation of $\Phi$.

\section{3. $\rho$-Contractive homomorphism}

A bounded linear operator $T$ on $H$ is said to be of class $C_{\rho}$ if there exists a unitary operator $U$ (called a unitary $\rho$-dilation) on a Hilbert space $K \supset H$ such that $T^{n}=\rho P U^{n} \mid H$ for $n=1,2, \cdots$ where $P$ is an orthogonal projection from $K$ to $H$. B.Sz.-Nagy [9] showed that if $T$ is a contraction then it is of class $C_{1}$. If the numerical radius of $T$ is less than equal to one then it is of class $C_{2}$ [3]. If $T$ is of class $C_{\rho}$ then $\|T\| \leq \max (1, \rho)$. 
Suppose $\Phi$ is a unital algebra homomorphism of $A$ in $L(H)$ and $0<\rho<\infty$. When $\Phi(f)$ is of class $C_{\rho}$ for any $f$ in $A$ with $\|f\|_{\infty} \leq 1, \Phi$ is called a $\rho$-contractive homomorphism of $A$. A 1-contractive homomorphism is equivalent to a contractive homomorphism. A 2-contractive homomorphism $\Phi$ is equivalent to that

$$
\sup _{\substack{f \in A \\\|f\|_{\infty} \leq 1}} \sup _{\substack{y \in H \\\|y\|=1}}|\langle\Phi(f) y, y\rangle|=1
$$

If $\Phi$ is $\rho$-contractive then $\|\Phi\| \leq \max (1, \rho)$. We will study Problem (II- $b$ ) when $\Phi$ is $\rho$-contractive.

A unital contractive homomorphism $v \rightarrow \tilde{\Phi}(v)$ on a Hilbert space $K$ is called a $\rho$-dilation of the unital bounded homomorphism $f \rightarrow \Phi(f)$ of $A$ on $H$ if $H$ is a Hilbert subspace of $K$ and

$$
\Phi(f)=\rho P \tilde{\Phi}(f) \mid H \quad\left(f \in A_{\tau}\right)
$$

where $P$ is the orthogonal projection of $K$ onto $H, A_{\tau}$ is the kernel of $\tau$ in $M(A)$ and $0<\rho<\infty$.

If $\Phi$ has a $\rho$-dilation then $\Phi$ is a unital completely bounded map. However the converse is not true even for the disc algebra $A$ and $\operatorname{dim} H=2$. This may be well known. Suppose $T=\left[\begin{array}{rr}1 & 1 \\ 0 & -1\end{array}\right]$, then $f \rightarrow \Phi(f)=f(T)$ is a unital completely bounded homomorphism but it has not a $\rho$-dilation for any $\rho$ [15]. If $\Phi$ is a unital completely bounded homomorphism, then $\Phi / \rho$ is completely contractive but it is not unital. However if $\Phi$ has a $\rho$-dilation then the following is true.

PropositTon 3.1. For a unital bounded homomorphism $\Phi, \Phi$ has a $\rho$-dilation with respect to $\tau$ if and only if $\Phi(f)=\rho \Phi_{0}(f)\left(f \in A_{\tau}\right)$ where $\Phi_{0}$ is a unital completely contractive map on $A$, equivalently $\Phi_{0}$ has a contractive dilation.

Proof. For the 'only if' part, put $\Phi_{0}(f)=P \tilde{\Phi}(f) \mid H(f \in A)$. Then $\Phi_{0}$ has a contractive dilation $\tilde{\Phi}$ and hence it is completely contractive on $A$. The 'if' part follows from a theorem of W.Arveson (cf.[18, Corollary 6.7]).

\section{4. $\delta$-homomorphism}

For $-\infty<\delta<1, \Phi$ is called a $\delta$-homomorphism for $\tau$ in $M(A)$ if $\Phi$ is a unital algebra homomorphism of $A$ and $\operatorname{Re} \Phi(f) \geq 0$ whenever $f$ in $A, \tau(f)=1$ and Ref $\geq \delta$. In this section we show that $\Phi$ is a $\delta$-homomorphism for some $\tau$ if and only if $\Phi$ is a $\rho=1 /(1-\delta)$-contractive homomorphism.

Proposition 4.1. If a unital algebra homomorphism $\Phi$ has a $\rho$-dilation for $\tau$, then $\Phi$ is a $\delta=\left(1-\frac{1}{\rho}\right)$-homomorphism for $\tau$.

Proof. Suppose that $\Phi(h)=\rho P \tilde{\Phi}(h) \mid H$ for $h \in A_{\tau}$. If $f \in A, \tau(f)=1$ and Ref $\geq 1-\frac{1}{\rho}$, since $\tau(f-\tau(f))=0$,

$$
\Phi(f)-\tau(f) I=\rho P \tilde{\Phi}(f) \mid H-\rho \tau(f) I
$$

and so

$$
\Phi(f)=\rho\left\{P \tilde{\Phi}(f) \mid H+\tau(f)\left(\frac{1}{\rho}-1\right) I\right\} .
$$


Since $\operatorname{Ref} \geq 1-\frac{1}{\rho}, \tilde{\Phi}(\operatorname{Ref}) \geq 1-\frac{1}{\rho}$ and so

$$
\operatorname{Re} \Phi(f) \geq \rho\left\{P \tilde{\Phi}(\operatorname{Ref}) \mid H+\left(\frac{1}{\rho}-1\right) I\right\} \geq 0
$$

Proposition 4.2. Suppose $\delta \neq 0$. $\Phi$ is a $\delta$-homomorphism for $\tau$ if and only if for any $h$ in $A_{\tau}$ with $\|h\|_{\infty}<1$,

$$
|\langle\Phi(h) y, y\rangle| \leq \frac{1}{2|\delta|}\|y\|^{2}+\frac{2 \delta-1}{2|\delta|}\|\Phi(h) y\|^{2} \quad(y \in H)
$$

Proof. Suppose $\Phi$ is a $\delta$-homomorphism for $\tau$. Put

$$
f=(1-\delta) \frac{1+h}{1-h}+\delta
$$

where $h \in A_{\tau}$ and $\|h\|_{\infty}<1$ then $f \in A, R e f \geq \delta$ and $\tau(f)=1$. For $x \in H$, put $x=(I-\Phi(h)) y$ then

$$
\begin{aligned}
& \operatorname{Re}\langle\Phi(f) x, x\rangle \\
& =(1-\delta) \operatorname{Re}\left\langle\frac{I+\Phi(h)}{I-\Phi(h)} x, x\right\rangle+\delta\|x\|^{2} \\
& =(1-\delta) \operatorname{Re}\langle(I+\Phi(h)) y,(I-\Phi(h)) y\rangle+\delta\langle(I-\Phi(h)) y,(I-\Phi(h)) y\rangle \\
& =(1-\delta)\left(\|y\|^{2}-\|\Phi(h) y\|^{2}\right)+\delta\left(\|y\|^{2}+\|\Phi(h) y\|^{2}-2 \operatorname{Re}\langle\Phi(h) y, y\rangle\right) \\
& =\|y\|^{2}+(2 \delta-1)\|\Phi(h) y\|^{2}-2 \delta\langle\Phi(h) y, y\rangle .
\end{aligned}
$$

By hypothesis on $\Phi$,

$$
2 \delta \operatorname{Re}\langle\Phi(h) y, y\rangle \leq\|y\|^{2}+(2 \delta-1)\|\Phi(h) y\|^{2}
$$

and so

$$
|\langle\Phi(h) y, y\rangle| \leq \frac{1}{2|\delta|}\|y\|^{2}+\frac{2 \delta-1}{2|\delta|}\|\Phi(h) y\|^{2} .
$$

The proof is reversible. In fact, if $f \in A, \operatorname{Re} f \geq \delta$ and $\tau(f)=1$ then

$$
f=(1-\delta) \frac{1+h}{1-h}+\delta
$$

for some $h \in A_{\tau}$ with $\|h\|_{\infty} \leq 1$. Hence if we put for $0<\varepsilon<1$

$$
f_{\varepsilon}=(1-\delta) \frac{1+\varepsilon h}{1-\varepsilon h}+\delta
$$

then $f_{\varepsilon} \rightarrow f$ uniformly as $\varepsilon \rightarrow 1,\|\varepsilon h\|_{\infty} \leq 1$ and $\tau\left(f_{\varepsilon}\right)=1$. Since $\operatorname{Re}\left\langle\Phi\left(f_{\varepsilon}\right) x, x\right\rangle \geq$ 0 , as $\varepsilon \rightarrow 1, \operatorname{Re}\langle\Phi(f) x, x\rangle \geq 0$ for any $x \in H$.

TheOREM 4.3. $\Phi$ is a $\delta$-homomorphism for some (or any) $\tau$ in $M(A)$ if and only if $\Phi$ is a $\rho=1 /(1-\delta)$-contractive homomorphism.

Proof. [16, Theorem 2] and Proposition 4.2 imply the theorem, or we can show this by the proof of Proposition 4.2 and [11, Theorem 11.1]. 


\section{Condition $A$}

In this section, we show that a $\rho$-contractive homomorphism has a $\rho$-dilation when $A$ is a Dirichlet algebra. This is a generalization of a theorem of C.Foias and I.Suciu [7] for $\rho=1$ and a theorem of B.Sz.Nagy and C.Foias (cf. [11]) for the disc algebra. They give solutions for Problem (II-b).

THEOREM 5.1. Let $A$ be a Dirichlet algebra and $0<\rho<\infty$. If $\Phi$ is a $\rho$ contractive homomorphism of $A$ in $L(H)$ then for any $\tau$ in $M(A)$ it has a $\rho$-dilation.

Proof. Put $\Phi^{\prime}(h)=\frac{1}{\rho} \Phi(h)-\tau(h)\left(\frac{1}{\rho}-1\right) I$ for $h \in A$. By Theorem 4.3 if $\Phi$ is $\rho$-contractive then $\Phi$ is a $\delta=\left(1-\frac{1}{\rho}\right)$ - homorphism for any $\tau \in M(A)$. Hence if $\operatorname{Re} h \geq 0$ then $\operatorname{Re} \Phi^{\prime}(h) \geq 0$. If we extend $\Phi^{\prime}$ to $\tilde{\Phi}: A+\bar{A} \rightarrow L(H)$ by $\tilde{\Phi}(f+\bar{g})=\Phi^{\prime}(f)+\Phi^{\prime}(g)^{*}$, then $\tilde{\Phi}: C(X) \rightarrow L(H)$ is positive because $A$ is a Dirichlet algebra. By the dilation theorem of M.A.Naimark (cf. [21, Theorem 7.5]) there exists a Hilbert space $K$, an orthogonal projection $P: K \rightarrow H$ and a multiplicative linear map $u \rightarrow \tilde{\Phi}(u)$ of $C(X)$ in $L(K)$, which satisfies $\tilde{\Phi}(1)=$ $I_{K},\|\tilde{\Phi}(u)\| \leq\|u\|_{\infty}, u \in C(X)$ and $\Phi^{\prime}(f)=P \tilde{\Phi}(f) \mid H$ for $f \in A$. If $f \in A_{\tau}$ then $\Phi^{\prime}(f)=\frac{1}{\rho} \Phi(f)$ and so

$$
\Phi(f)=\rho P \tilde{\Phi}(f) \mid H
$$

Proposition 5.2. Let $A$ be an arbitrary uniform algebra and $0<\rho<\infty$. Suppose $\Phi$ is a $\rho$-contractive homomorphism of $A$ in $L(H)$. If $A / \operatorname{ker} \Phi$ is isometrically isomorphic to $\mathcal{A} / \mathcal{J}$ where $\mathcal{A}$ is a Dirichlet algebra on some compact Hausdorff space $Y$ and $\mathcal{J}$ is a closed ideal in $\mathcal{A}$, then $\Phi$ has a $\rho$-dilation for any $\tau$ in $M(A)$ with $\tau=0$ on $\operatorname{ker} \Phi$.

PROOF. Let $\phi$ be an isometric isomorphism from $\mathcal{A} / \mathcal{J}$ onto $A /$ ker $\Phi$. For each $f \in A$, we will write $\phi(f+\mathcal{J})=\phi(f)+\operatorname{ker} \Phi$ where $\phi(f) \in \mathcal{A}$. Moreover we will write $\Phi$ again for the map : $f+\operatorname{ker} \Phi \rightarrow \Phi(f)$. Put $\Psi=\Phi \circ \phi$, then $\Psi$ is a unital homomorphism of $\mathcal{A} / \mathcal{J}$ in $L(H)$. We will write $\Psi$ again for the map $: f \rightarrow \Psi(f+\mathcal{J})$, then $\mathcal{J}=\operatorname{ker} \Psi$. Since we may assume that $\tau$ is a complex homomorphism on $A / \operatorname{ker} \Phi$ by [8, Theorem 6.2 in Chapter I], $\tau \circ \phi$ is a complex homomorphism on $\mathcal{A} / \mathcal{J}$ and so we may assume that $\tau \circ \phi \in M(A)$. If $f \in A_{\tau \circ \phi}$. and $\|f\|_{\infty} \leq 1$, then $\phi(f) \in A_{\tau}$ and $\|\phi(f)+\mathcal{J}\| \leq 1$. By hypothesis, $\Phi(\phi(f))$ is of class $C_{\rho}$ and so $\Psi(f)=\Phi \circ \phi(f)$ is of class $C_{\rho}$ for $f \in \mathcal{A}_{\tau \circ \phi}$ with $\|f\|_{\infty} \leq 1$. Hence $\Psi$ is a $\rho$-contractive homomorphism of $\mathcal{A}$ in $L(H)$ with respect to $\tau \circ \phi$. Since $\mathcal{A}$ is a Dirichlet algebra, by Proposition 3.1 and Theorem 5.1 $\Psi=\rho \Psi_{0}$ on $\mathcal{A}_{\tau \circ \phi}$ where $\Psi_{0}$ is a unital completely contractive map on $\mathcal{A}$. Put $\Phi_{0}=\Psi_{0} \circ \phi^{-1}$ then $\Phi_{0}$ is a unital completely contractive map on $A$ and $\Phi=\rho \Phi_{0}$ on $A_{\tau}$. Proposition 3.1 implies the theorem.

Let $A$ be a $n$-hypo-Dirichlet algebra and let $N_{\tau}$ be the set of all representing measures of $\tau$ in $M(A)$. Then $\operatorname{dim} N_{\tau}=n$ and there exists a core measure $m$ of $N_{\tau}$ (cf. [8, p106]). Then by [8, Theorem 5.1 in Chapter IV], there is a constant $c>0$ such that $\nu \leq c m$ for all $\nu$ in $N_{\tau}$. Hence if $h$ is the Radon-Nikodym derivative of $\nu$ with respect to $m$ then $\nu=h d m$. Set $N_{\tau}^{m}=\{h: \nu=h d m$ and 
$\left.\nu \in N_{\tau}\right\}$, then $N_{\tau}^{m}$ is a subset of $L^{\infty}(m)$. Thus $N_{\tau}^{m}$ can be considered as a subset of $L^{\infty}(m)$. Many important $n$-hypo-Dirichlet algebras satisfy a natural condition on $N_{\tau}^{m}: N_{\tau}^{m} \subset C(X)$. The author showed. [13] that if $N_{\tau}^{m} \subset C(X)$ then a unital contractive homomorphism $\Phi$ of $A$ has a $\rho$-dilation with respect to $\tau$. It is a long standing open question whether we can choose $\rho=1$. The motivation of our study in this paper is in this open question. The following Proposition 5.4 implies that if $\Phi$ is $\rho$-contractive for enough small $\rho>0$ then $\Phi$ has a 1-dilation.

LEMMA 5.3. Let $A$ be a n-hypo-Dirichlet algebra and $N_{\tau}^{m} \subset C(X)$. Then there exists a positive linear map $T$ from $C(X)$ to $[A+\bar{A}]$ such that $T(f)=f\left(f \in A_{\tau}\right)$ and $T(1)$ is a positive constant $\geq 1$.

Proof. This is proved in the proof of [13, Theorem].

Proposition 5.4. Let $A$ be a n-hypo-Dirichlet algebra and $N_{\tau}^{m} \subset C(X)$ for some $\tau$ in $M(A)$ where $m$ is a core measure of $N_{\tau}$. If $\Phi$ is a $\rho$-contractive homomorphism of $A$ in $L(H)$ then it has a $\rho^{\prime}$-dilation where $\rho^{\prime}=\rho T(1)$ and $T$ is a map in Lemma 5.3.

Proof. Suppose $\Phi$ is $\rho$-contractive. Then $\Phi$ is a $\delta=\left(1-\frac{1}{\rho}\right)$-homomorphism for $\tau$ by Theorem 4.3. Put

$$
\Phi^{\prime}(f)=\frac{1}{\rho} \Phi(f)-\tau(f)\left(\frac{1}{\rho}-1\right) I \quad(f \in A),
$$

then by the proof of Theorem 5.1, there exists an extension $\tilde{\Phi}^{\prime}$ of $\Phi^{\prime}$ on $[A+\bar{A}]$ and $\tilde{\Phi}^{\prime}$ is positive on it. Then $T(1)^{-1} \tilde{\Phi}^{\prime} \circ T$ is a positive map from $C(X)$ to $L(H)$ and $T(1)^{-1} \tilde{\Phi}^{\prime} \circ T(1)=I$. By the dilation theorem of M.A.Naimark (cf. [21, Theorem 7.5] there exists a Hilbert space $K$, an orthogonal projection $P$ from $K$ to $H$ and a multiplicative linear map $u \rightarrow \tilde{\Phi}(u)$ of $C(X)$ in $L(K)$, which satisfies $\tilde{\Phi}(1)=I_{K},\|\tilde{\Phi}(u)\| \leq\|u\|_{\infty}, u \in C(X)$ and $\tilde{\Phi}^{\prime} \circ T(u)=T(1) P \tilde{\Phi}(u) \mid H$. Because $T(f)=f\left(f \in A_{\tau}\right)$,

$$
\Phi(f)=\rho T(1) P \tilde{\Phi}(f) \mid H \quad\left(f \in A_{\tau}\right) .
$$

Suppose $\rho^{\prime}=\rho T(1)$.

\section{Condition on $\Phi$}

In this section, under conditions on $\Phi$ we consider Problem II. We consider $\rho$-contractive homomorphisms when $A / k e r \Phi$ is of two dimension. The author and the late K.Takahashi [13] showed that $\Phi$ has a 1-dilation when $\rho=1$. We generalize it to any $\rho$. Proposition 6.3 is a generalization of a result of G.Misra [8] which was shown for a rational uniform algebra on the complex plane and $\rho=1$.

For $x, y$ in $M(A)$ and a bounded point derivation $\delta$ at $x$, let

$$
\sigma_{A}(x, y)=\sup \left\{|f(y)| ; f(x)=0, f \in A \text { and }\|f\|_{\infty} \leq 1\right\}
$$

and

$$
\omega_{A}(x, \delta)=\sup \left\{|\delta(f)| ; f(x)=0, f \in A \text { and }\|f\|_{\infty} \leq 1\right\} .
$$

THEOREM 6.1. Let $A$ be an arbitrary uniform algebra. If $\Phi$ satisfies one of the following conditions (1), (2) and (3), then $\Phi$ is completely bounded and $\|\Phi\|=$ $\|\Phi\|_{c b}$.

$$
\text { 1. }\left\|\Phi(f)^{2}\right\|=\|\Phi(f)\|^{2} \quad(f \in A)
$$


2. $E$ is an interpolation set in $X$ and $\operatorname{ker} \Phi=\{f \in A ; f=0$ on $E\}$

3. $\|\Phi\| \leq 1, \operatorname{ker} \Phi=\{f \in A ; f=0$ on $E\}$ for some finite set $E \subset M(A)$ and $\sigma_{A}(x, y)=1$ for any $x, y$ in $E$ with $x \neq y$.

Proof. (1) If $\left\|\Phi(f)^{2}\right\|=\|\Phi(f)\|^{2}(f \in A)$ then the closure of $\Phi(A)$ is regarded as a uniform algebra. By [18, Theorem 3.8], $\|\Phi\|=\|\Phi\|_{c b}$.

(2) Since $E$ is an interpolation set in $X, A / \operatorname{ker} \Phi$ is isometrically isomorphic to a subalgebra of $C(E)$ and hence $\Phi(A) \subseteq C(E)$. Again by [18, Theorem 3.8], $\|\Phi\|=\|\Phi\|_{c b}$.

(3) Since $E=\left\{x_{1}, \cdots, x_{n}\right\}$ is a finite set, $\operatorname{dim} A / \operatorname{ker} \Phi=n<\infty$ and $\Phi(A)=$ $\left\{\sum_{j=1}^{n} a_{j} P_{j} ; a_{j} \in \mathbf{C}, P_{i} P_{j}=\delta_{i j} P_{j}\right.$ and $\left.j=1, \cdots, n\right\}$. Suppose $\Phi\left(f_{j}\right)=P_{j}$ and $f_{j} \in$ $A$. Then $f_{i} f_{j}=\delta_{i j} f_{j}$ and $f_{i}\left(x_{j}\right)=\delta_{i j}$. Since $\sigma_{A}\left(x_{i}, x_{j}\right)=\delta_{i j}$, there exist $\left\{g_{n}^{(i)}\right\}_{n=1}^{\infty}$ in $A$ such that $g_{n}^{(i)}\left(x_{i}\right) \rightarrow 1(n \rightarrow \infty), g_{n}^{(i)}\left(x_{j}\right)=0(j \neq i)$ and $\left\|g_{n}^{(i)}\right\|_{\infty} \leq 1$. Since $A / \operatorname{ker} \Phi=\left\{\sum_{i=1}^{n} a_{i} f_{i}+\operatorname{ker} \Phi ; a_{i} \in \mathbf{C}\right.$ and $\left.i=1, \cdots, n\right\}, g_{n}^{(i)}-a_{i n} f_{i} \in \operatorname{ker} \Phi$ and $\Phi\left(g_{n}^{(i)}\right)=a_{i n} P_{i}$. Since $\Phi$ is contractive, $\left|a_{i n}\right||| P_{i} \| \leq 1$ and so $\left\{a_{i n}\right\}$ is bounded. Hence there exists a subsequence $\left\{a_{i n(j)}\right\}$ such that $a_{i n(j)} \rightarrow a_{i}$ as $j \rightarrow \infty$ for each $i$. Then $\lim _{j \rightarrow \infty} g_{n(j)}^{(i)}-a_{i} f_{i} \in \operatorname{ker} \Phi$ and $\lim _{j \rightarrow \infty} g_{n(j)}^{(i)}\left(x_{i}\right)=1$. Therefore $a_{i}=1$ and $\left\|f_{i}+\operatorname{ker} \Phi\right\| \leq 1$, and $P_{i}$ is selfadjoint for $i=1, \cdots, n$ because $\Phi$ is contractive. Thus $\Phi(A)$ is a commutative $C^{*}$-algebra. By [18, Theorem 3.8], $\|\Phi\|=\|\Phi\|_{c b}$.

TheOREM 6.2. Suppose $\Phi$ is a $\rho$-contractive homomorphism of $A$. If $A / \operatorname{ker} \Phi$ is of two dimension then $\Phi$ has a $\rho$-dilation for any $\tau$ in $M(A)$ with $\tau=0$ on $\operatorname{ker} \Phi$.

Proof. Suppose ker $\Phi=\{f \in A ; f(x)=f(y)=0\}$ where $x, y \in M(A)$ with $x \neq y$. By $[13$, Lemma 1 and its proof $]$,

$$
\Phi(f)=\left(\begin{array}{cc}
f(x) I_{H_{1}} & (f(x)-f(y)) C \\
0 & f(y) I_{H_{2}}
\end{array}\right) \text { on } H=H_{1} \oplus H_{2}
$$

for all $f \in A$ where $C$ is a bounded linear operator from $H_{2}$ to $H_{1}$, and

$$
A / \operatorname{ker} \Phi=\left\{f(x) f_{1}+f(y) f_{2}+\operatorname{ker} \Phi ; f \in A\right\}
$$

where $f_{1}(x)=f_{2}(y)=1$ and $f_{1}(y)=f_{2}(x)=0$. By [13, Lemma 3], if $\|C\|^{2}+1=$ $1 / \sigma_{A}(x, y)^{2}$ then $\|\Phi(f)\|=\|f+\operatorname{ker} \Phi\|$ for all $f \in A$.

For any $x, y \in M(A)$, there exist a Dirichlet algebra $\mathcal{A}$ and $s, t \in M(\mathcal{A})$ such that $\sigma_{A}(x, y)=\sigma_{\mathcal{A}}(s, t)$. In fact, we can choose the disc algebra $\mathcal{A}$. Suppose

$$
\Psi(F)=\left(\begin{array}{cc}
F(s) I_{H_{1}} & (F(s)-F(t)) B \\
0 & F(t) I_{H_{2}}
\end{array}\right)
$$

for all $F \in \mathcal{A}$ where $B$ is a bounded linear operator from $H_{2}$ to $H_{1}$. Then

$$
\mathcal{A} / \operatorname{ker} \Psi=\left\{F(s) F_{1}+F(t) F_{2}+\operatorname{ker} \Psi ; F \in \mathcal{A}\right\}
$$

where $F_{1}(s)=F_{2}(t)$ and $F_{1}(t)=F_{2}(s)$, and $\|\Phi(f)\|=\|\Psi(F)\|$ whenever $f(x)=$ $F(s)$ and $f(y)=F(t)$, and $B=C$. If $B=C$ and $\|C\|^{2}+1=1 / \sigma_{A}(x, y)^{2}$, then $\|B\|^{2}+1=1 / \sigma_{A}(s, t)^{2}$ and so $\|f+\operatorname{ker} \Phi\|=\|\Phi(f)\|=\|\Psi(f)\|=\|f+\operatorname{ker} \Psi\|$. Hence for given $\Phi$, we can find a unital homomorphism $\Psi$ on $\mathcal{A}$ such that $A / \operatorname{ker} \Phi \cong$ $\mathcal{A} / \operatorname{ker} \Psi$. By Proposition 5.2, $\Phi$ has a $\rho$-dilation for any $\tau \in M(A)$ with $\tau=0$ on $\operatorname{ker} \Phi .$. If $\operatorname{ker} \Phi$ is not the above form, then $\operatorname{ker} \Phi=\{f \in A ; f(x)=\delta(f)=0\}$ 
where $x \in M(A)$ and $\delta$ is a bounded point derivation at $x$. By [13, Lemma 1 and its proof],

$$
\Phi(f)=\left(\begin{array}{cc}
f(x) I_{H_{1}} & \delta(f) C \\
0 & f(x) I_{H_{2}}
\end{array}\right) \text { on } H=H_{1} \oplus H_{2}
$$

for all $f \in A$ where $C$ is a bounded linear operator from $H_{2}$ to $H_{1}$, and

$$
A / \operatorname{ker} \Phi=\left\{f(x) 1+\delta(f) f_{0}+\operatorname{ker} \Phi ; f \in A\right\}
$$

where $f_{0}(x)=0$ and $\delta\left(f_{0}\right)=1$. By [13,Lemma 3], if $\|C\|=1 / \omega_{A}(x, \delta)$ then $\|\Phi(f)\|=\|f+\operatorname{ker} \Phi\|$ for all $f \in A$. As in the first part of the proof, by Proposition 5.2 we can show that $\Phi$ has a $\rho$-dilation for any $\tau \in M(A)$.

If $\operatorname{dim} H=2$, then an algebra homomorphism $\Phi$ has the following form :

$$
\Phi_{1}(f)=\left(\begin{array}{cc}
f(x) & c(f(x)-f(y)) \\
0 & f(y)
\end{array}\right)
$$

where $x, y \in M(A)$ and $x \neq y$ or

$$
\Phi_{2}(f)=\left(\begin{array}{cc}
f(x) & c \delta(f) \\
0 & f(x)
\end{array}\right)
$$

where $x \in M(A)$ and $\delta$ is a bounded point derivation at $x$.

Proposition 6.3. Suppose $\Phi$ is a unital bounded homomorphism of $A$ in $L(H)$ and $\operatorname{dim} H=2$.

1. When $\Phi=\Phi_{1}, \Phi$ is a $\rho$-contractive homomorphism if and only if

$$
\begin{aligned}
& \left(1+|c|^{2}\right)|\rho \zeta(f(x)-f(y))|^{2} \\
& \quad \leq\left.\left.|\{\rho+(1-\rho) \overline{f(x)} \bar{\zeta}\}\{\rho+(1-\rho) f(y) \zeta\}-\overline{f(x)} f(y)| \zeta\right|^{2}\right|^{2}
\end{aligned}
$$

for any $f \in A$ with $\|f\|_{\infty} \leq 1$ and any $\zeta \in D$.

2. When $\Phi=\Phi_{2}, \Phi$ is a $\rho$-contractive homomorphism if and only if

$$
\begin{aligned}
& |c|^{2}|\delta(f)|^{2} \\
& \quad \leq(\rho-2)|f(x)|^{2}+2(1-\rho)|f(x)|+\rho
\end{aligned}
$$

for any $f \in A$ with $\|f\|_{\infty} \leq 1$.

Proof. The author and Okubo [15] gave a necessary and sufficient condition for that a triangle $2 \times 2$ matrix is of class $C_{\rho}$. By [15, Theorem] $\Phi_{1}(f)$ is of class $C_{\rho}$ if and only if

$$
\begin{aligned}
& |c|^{2}|f(x)-f(y)|^{2}+|f(x)-f(y)|^{2} \\
& \quad \leq \inf _{\zeta \in D}\left|\frac{\{\rho+(1-\rho) \overline{f(x)} \bar{\zeta}\}\{\rho+(1-\rho) f(y) \zeta\}-\overline{f(x)} f(y)|\zeta|^{2}}{\rho \zeta}\right|^{2}
\end{aligned}
$$

and by $\left[15\right.$, Remark] $\Phi_{2}(f)$ is of class $C_{\rho}$ if and only if

$$
|c|^{2}|\delta(f)|^{2} \leq(\rho-2)|f(x)|^{2}+2(1-\rho)|f(x)|+\rho .
$$

In Proposition 6.3, suppose $\rho=1 . \Phi=\Phi_{1}$ is a 1-contractive homomorphism if and only if $|c|^{2} \leq\left(1-|f(x)|^{2}\right)\left(1-|f(y)|^{2}\right) /|f(x)-f(y)|^{2}$ for any $f \in A$ with $\|f\|_{\infty} \leq 1$. This implies [9, Theorem 1.1]. $\Phi=\Phi_{2}$ is a 1-contractive homomorphism if and only if $|c|^{2} \leq\left(1-|f(x)|^{2}\right) /|\delta(f)|^{2}$ for any $f \in A$ with $\|f\|_{\infty} \leq 1$. Suppose $\rho=2 . \Phi=\Phi_{1}$ is a 2-contractive homomorphism if and only if $1+|c|^{2} \leq$ 
$\inf _{\zeta \in D}\left|\frac{2-(\overline{f(x)} \bar{\zeta}+f(x) \zeta)}{\zeta(f(x)-f(y))}\right|^{2}$ for any $f \in A$ with $\|f\|_{\infty} \leq 1 . \Phi=\Phi_{2}$ is a 2-contractive homomorphism if and only if $|c|^{2} \leq 2(1-|f(x)|) /|\delta(f)|^{2}$ for any $f \in A$ with $\|f\|_{\infty} \leq 1$.

\section{References}

[1] J.Agler Rational dilation on an annulus, Ann. of Math., 121 (1985), 537-564.

[2] T.Ando On a pair of commutative contractions, Acta Sci. Math., 24 (1963), 88-90.

[3] C.A.BergerA strange dilation theorem, Notices Amer. Math. Soc., 12 (1965), 590.

[4] F.Bonsal and J.Duncan Complete normed algebras, Springer-Verlag, New York and Berlin, 1973.

[5] Che-Chen Chu Finite dimensional representation of a function algebra, $\mathrm{Ph} . \mathrm{D}$. thesis in the University of Houston, 1992.

[6] R.G.Douglas and V.I.Paulsen Completely bounded maps and hypo-Dirichlet algebras, Acta Sci. Math., 50 (1986), 143-157.

[7] C.Foias and I.Suciu Szegö-measures and spectral theory in Hilbert spaces, Rev. Roum. Math. Pures. et Appl. 11 (1966), 147-159.

[8] T.Gamelin Uniform Algebras, Prentice-Hall, Englewood Cliffs, New Jersey, 1969.

[9] G.Misra Curvature inequalities and extremal properties of bundle shifts, J. Operator Theory 11 (1984), 305-317.

[10] B.Sz.-Nagy Sue les contractions de e'spase de Hilbert, Acta Sci. Math. 15 (1953), 87-92.

[11] B.Sz.-Nagy and C.Foias Harmonic Analysis of Operators on Hilbert Space, North Holland, New York, 1970.

[12] T.Nakazi A spectral dilation of some non-Dirichlet algebra, Acta Sci. Math., 53 (1989), 119122

[13] T.Nakazi $\rho$-dilations and hypo-Dirichlet algebras, Acta Sci. Math., 56 (1992), 175-181.

[14] T.Nakazi and K.Takahashi Two-dimensional representations of uniform algebras, Proc. Amer. Math. Soc. 123 (1995), 2777-2784.

[15] T.Nakazi and K.Okubo $\rho$-Contraction and $2 \times 2$ matrix, Linear Algebra Appl. 283 (1998), 165-169.

[16] T.Nakazi and K.Okubo Generalized numerical radius and unitary $\rho$-Dilation, to appear in Math. Japonica.

[17] S.Parrot Unitary dilations for commuting contractions, Pacific J. Math., 34 (1970), 481-490.

[18] V.I.Paulsen Completely Bounded Maps and Dilations, Pitman Research Notes in Math. 146, Longman, Wiley, New York, 1986.

[19] G.Pisier A polynomially bounded operator on Hilbert space which is not similar to a contraction, J.Amer. Math. Soc. 10 (1997), 351-369.

[20] H.Radjavi and P.Rosenthal Invariant Subspaces, Springer-Verlag, New York, 1973.

[21] I.Suciu Function Algebras, Editura Academiej Republicii Socialiste Romania, Bucuresti, 1973.

Department of Mathematics, HokKaido University, Sapporo 060-0810, JaPAN

E-mail address: nakazi@math.sci.hokudai.ac.jp 